\title{
Estimating signal and noise using a random array ${ }^{\text {a) }}$
}

\author{
Melvin J. Hinich \\ Virginia Polytechnic Institute and State University, Blacksburg, Virginia 24061 \\ (Received 6 August 1981; accepted for publication 30 September 1981)
}

\begin{abstract}
This paper presents approximations for the rms error of the maximum likelihood estimator of the direction of a plane wave incident on a random array. The sensor locations are assumed to be realizations of independent, identically distributed random vectors. The second part of the paper presents an asymptotically unbiased estimator of the noise wavenumber spectrum from random array data.
\end{abstract}

PACS numbers: $43.60 . \mathrm{Cg}, 43.60 . \mathrm{Gk}$

\section{INTRODUCTION}

Sonobuoy fields are used to detect submarines. Thorn et al. ${ }^{1}$ have proposed that the signals from randomly deployed sonobuoys be coherently combined to make acoustic measurements. They present the expected value and variance of the pattern function, and the distribution of the directivity index of a three-dimensional random array. In their model, the sensor locations are observed realizations of random variables that may be correlated and have different distributions. They define an array to be totally random if the sensor locations are realizations of independent, identically distributed random variables. Several stochastic properties of the side-lobe pattern of a totally random array are given by Steinberg. ${ }^{2}$

The ratio of the peak side lobe to the mainlobe and the directivity index of an array system are measures of its ability to perform its tasks. The generic signal processing tasks of an array system are (1) detecting and estimating parameters of coherent wave signals that impinge on the array; (2) resolving multiple wave signals; (3) estimating range, bearing, or velocity of a source that generates the detected signal; and (4) estimating the frequency-wavenumber spectrum of the ambient noise field. This description of system tasks emphasizes the statistical nature of the problem of measuring performance, especially for random arrays.

This paper presents approximations for the meansquare error of the maximum likelihood estimator of the bearing of a plane wave impinging on a random array from a distant source. The second part deals with estimating the ambient noise's wavenumber spectrum.

\section{RANDOM PLANAR ARRAYS}

Consider a planar array of $M$ sensors where the sensor locations $\left\{\left(x_{k}, y_{k}\right)\right\}$ are realizations of independent, identically distributed random variables $\left\{\left(X_{k}, Y_{k}\right)\right\}$. Assume for simplicity that the signal is a single frequency plane wave plus stationary, zero mean, Gaussian noise. Let $\theta_{0}$ denote the wave's direction of arrival with respect to the $x$ axis. This angle is the source bearing if the medium is horizontally homogeneous. Let $\omega_{0}, \lambda_{0}$, and $\boldsymbol{A}$ denote the wave's frequency, wavelength, and complex amplitude, respectively. The signal at the $k$ th sensor is

a) This work was supported by the Office of Naval Research (Statistics and Probability Program) under contract.

$$
\begin{aligned}
s\left(t, x_{k}, y_{k}\right)= & A \exp \left[i\left(\omega_{0} t-\kappa_{x} x_{k}-\kappa_{y} y_{k}\right)\right] \\
& +\epsilon\left(t, x_{k}, y_{k}\right),
\end{aligned}
$$

where $\kappa_{x}=\left(2 \pi / \lambda_{0}\right) \cos \theta_{0}$ and $\kappa_{y}=\left(2 \pi / \lambda_{0}\right) \sin \theta_{0}$ are the $x$ and $y$ components of the wavenumber, and $\epsilon\left(t, x_{k}, y_{k}\right)$ is a realization of the noise field.

The correspondence between beamforming and frequency-wavenumber processing, and an approximation to the maximum likelihood (ML) estimator of $\theta_{0}$ have been presented in a previous paper. ${ }^{3}$ If

$$
\rho \sum_{k=1}^{k}\left(x_{k}-\bar{x}\right)^{2}
$$

and

$$
\rho \sum_{k=1}^{k}\left(y_{k}-\bar{y}\right)^{2}
$$

are large, where $\rho$ is the power signal-to-noise ratio in a narrow band about $\omega_{0}$ and

$$
\bar{x}=M^{-1} \sum_{k=1}^{n} x_{k},
$$

Levin ${ }^{4}$ shows that the root mean-square errors of the ML estimators of $\kappa_{x}$ and $\kappa_{y}$ are approximately

$$
\begin{aligned}
& \text { rmse } R_{x} \simeq\left(2 \rho \sum_{k=1}^{N}\left(x_{k}-\bar{x}\right)^{2}\right)^{-1 / 2}, \\
& \text { rmse } \hat{k}_{y} \simeq\left(2 \rho \sum_{k=1}^{N}\left(y_{k}-\bar{y}\right)^{2}\right)^{-1 / 2} .
\end{aligned}
$$

Moreover, the covariance is

$$
E\left(R_{x}-\kappa_{x}\right)\left(\hat{R}_{y}-\kappa_{y}\right) \simeq\left(2 \rho \sum_{k=1}^{k}\left(x_{k}-\bar{x}\right)\left(y_{k}-\bar{y}\right)\right)^{-1} .
$$

These expected values are conditional on a realized array geometry, i.e., they are ex-post the deployment of the array.

To approximate these errors, assume that $M$ is large. Since the sensors must lie in some closed and bounded set, the random variables $\left(X_{k}, Y_{k}\right)$ are bounded. Thus the central limit theorem implies that

$$
M^{-1} \sum_{k=1}^{N}\left(x_{k}-\bar{x}\right)^{2}=\sigma_{x}^{2}+O_{p}\left(M^{-1 / 2}\right)
$$

and

$$
M^{-1} \sum_{k=1}^{M}\left(y_{k}-\bar{y}\right)^{2}=\sigma_{y}^{2}+O_{p}\left(M^{-1 / 2}\right),
$$

where $\sigma_{x}^{2}$ and $\sigma_{y}^{2}$ are the variances of $X_{k}$ and $Y_{k}$, respec- 
tively, and $O_{p}\left(M^{-1 / 2}\right)$ means that for any $\epsilon>0$, there is a $B_{\epsilon}>0$ such that the error is bounded by $B_{\epsilon} M^{-1 / 2}$ with probability $1-\epsilon$. Thus the rms errors of $R_{x}$ and $R_{y}$ are approximately

$$
\begin{aligned}
& \text { rmse } R_{x} \simeq(2 \rho M)^{-1 / 2} \sigma_{x}^{-1}, \\
& \operatorname{rmse~} R_{y} \simeq(2 \rho M)^{-1 / 2} \sigma_{y}^{-1},
\end{aligned}
$$

for large $M$. The estimators are approximately uncorrelated if the coordinate system is rotated to make the covariance $\sigma_{x y}=0$ after rotation.

The maximum likelihood estimator of the bearing is $\hat{\theta}_{0}=\tan ^{-1}\left(R_{y} / R_{x}\right) \mathrm{rad}$. The linear approximation of $\tan ^{-1}\left(\hat{R}_{y} / \hat{R}_{x}\right)-\tan ^{-1}\left(\kappa_{y} / \kappa_{x}\right)$ is

$$
\left(1+\kappa_{y}^{2} \kappa_{x}^{-2}\right)^{-1}\left[\kappa_{x}^{-1}\left(R_{y}-\kappa_{y}\right)-\kappa_{y} \kappa_{x}^{-2}\left(\hat{R}_{x}-\kappa_{x}\right)\right] .
$$

Since $R_{x}$ and $R_{y}$ are approximately uncorrelated if $\sigma_{x y}=0$, it follows from (3) and (4) that when $\rho M \sigma_{x}^{2}$ and $\rho M \sigma_{y}^{2}$ are large,

$$
E\left(\hat{\theta}_{0}-\theta_{0}\right)^{2} \simeq\left(\lambda_{0} / 2 \pi\right)^{2}(2 \rho M)^{-1}\left(\sigma_{x}^{-2} \sin ^{2} \theta_{0}+\sigma_{y}^{-2} \cos ^{2} \theta_{0}\right) .
$$

Thus if $\sigma_{x}=\sigma_{y}=\sigma$, then from (5)

$$
\text { rmse } \hat{\theta}_{0} \simeq \lambda_{0}(2 \rho M)^{-1 / 2}(2 \pi \sigma)^{-1} \mathrm{rad} \text {. }
$$

For example, let $\sigma / \lambda_{0}=12, M=90$, and $\rho=\frac{1}{4}(-6 \mathrm{~dB})$. Then from (6), rmse $\hat{\theta}_{0}=0.11^{\circ}\left(1.98 \times 10^{-3} \mathrm{rad}\right)$. If $\sigma / \lambda_{0}$ $=100, M=40$, and $\rho=-10 \mathrm{~dB}$, then rmse $\hat{\theta}_{0}=0.03^{\circ}$.

Now suppose that $X_{k}$ and $Y_{k}$ are independent uniform variates whose range is $(0, L)$, i.e., the sensors are uniformly distributed on the square $\{0 \leqslant x \leqslant L, 0 \leqslant y \leqslant L\}$. Then $\sigma^{2}=L^{2} / 12$. Let us compare the rmse $\hat{\theta}_{0}$ of this random array with that of the square lattice array whose $M=N^{2}$ sensors are at the points $\{(j d, l d): j, l=1, \ldots, N\}$. If the length of the square's sides is $L$, then the sensor spacing is $d=L /(N-1)$.

From (2), (4), and (5), we only have to compare

$$
M^{-1} \sum\left(x_{k}-\bar{x}\right)^{2}=M^{-1} \sum\left(y_{k}-\bar{y}\right)^{2}
$$

with $\sigma^{2}$. Since

$$
\begin{aligned}
M^{-1} \sum_{k=1}^{M}\left(x_{k}-\bar{x}\right)^{2} & =M^{-1} d^{2} N \sum_{j=1}^{N}(j-\bar{j})^{2} \\
& =\frac{d^{2}(N-1)(N+1)}{12}=\frac{L^{2}}{12} \frac{N+1}{N-1} \\
& \simeq L^{2} / 12=\sigma^{2},
\end{aligned}
$$

expression (6) holds for the square lattice array. The approximate rmse of the maximum likelihood bearing estimator for a uniform random array on a square is equal to the approximate rmse $\hat{\theta}_{0}$ for a uniformly spaced lattice array on the same square.

\section{THREE-DIMENSIONAL RANDOM ARRAYS}

For a given coordinate system, let $x_{k}=\left(x_{k}, y_{k}, z_{k}\right)^{\prime}$ denote the vector location of the $k$ th sensor in a threedimensional array. Let $\theta_{0}$ denote the azimuth angle of propagation with respect to the $x$ axis, and let $\alpha_{0}$ denote the elevation angle with respect to the $z$ axis. Thus the signal at the $k$ th sensor is

$$
s\left(t, \mathbf{x}_{k}\right)=A \exp \left[i\left(\omega_{0} t-\kappa^{\prime} \mathbf{x}_{k}\right)\right]+\epsilon\left(t, \mathbf{x}_{k}\right),
$$

where $\kappa^{\prime}=\left(\kappa_{x}, \kappa_{y}, \kappa_{z}\right)$ is the vector of wavenumber components $\kappa_{x}=\left(2 \pi / \lambda_{0}\right) \cos \theta_{0}, \kappa_{y}=\left(2 \pi / \lambda_{0}\right) \sin \theta_{0}$, and $\kappa_{g}$ $=\left(2 \pi / \lambda_{0}\right) \cos \alpha_{0}$.

The correspondence between beamforming and frequency-wavenumber processing holds in three dimensions. The ML estimators of the wavenumber components are the $\kappa_{x}, \kappa_{y}$, and $\kappa_{z}$ that maximize

$$
\left|\sum_{j=1}^{N} \sum_{k=1}^{k} s\left(t_{j}, x_{k}, y_{k}, z_{k}\right) \exp \left[i\left(\kappa^{\prime} x_{k}-\omega_{0} t_{j}\right)\right]\right|^{2},
$$

where $N$ is the number of simultaneous discrete-time observations of the $M$ channels. ${ }^{5}$ The rms errors of $R_{x}$ and $R_{y}$ are approximated by (2), and

$$
\text { rmse } R_{z} \simeq\left(2 \rho \sum_{k=1}^{k}\left(z_{k}-\bar{z}\right)^{2}\right)^{-1 / 2} \text {. }
$$

Once again, the ML estimator of the source bearing is $\hat{\theta}_{0}=\tan ^{-1}\left(R_{y} / \hat{\kappa}_{x}\right)$, and thus (5) holds for a totally random three-dimensional array of $M$ sensors.

\section{ESTIMATING THE WAVENUMBER SPECTRUM}

Consider the problem of estimating the frequencywavenumber spectrum of the ambient, zero mean, Gaussian noise field around a random array. Since an $n$-dimensional array is not much harder to analyze than a linear array, let $\mathbf{x}_{k}=\left(x_{k 1}, \ldots, x_{k n}\right)^{\prime}$ denote the vector position of the $k$ th sensor with respect to a fixed coordinate system. Assume that the $\mathbf{x}_{k}$ are realizations of independent random vectors $\left\{\boldsymbol{X}_{k}=\left(X_{k 1}, \ldots\right.\right.$, $\left.\left.X_{k n}\right)^{\prime}\right\}$ that have a common continuous multivariate density $f(x)$. Rotate the coordinate system so that the covariance matrix of $\mathbf{X}_{k}$ is diagonal, and for simplicity let $\sigma_{1}^{2}=\ldots=\sigma_{n}^{2}=\sigma^{2}$, i.e., $\sigma^{2}$ is the variance of each $X_{h t}$ after rotation.

Let $\epsilon(t, \mathbf{x})$ be the noise at point $\mathbf{x}$ at time $t$. If the noise field is stationary in $t$ and $\mathbf{x}$, the covariance function $c_{\epsilon}(\tau, y)=E \epsilon(t+\tau, \mathbf{x}+\mathbf{y}) \epsilon(t, \mathbf{x})$ is independent of $t$ and x. The frequency-wavenumber spectrum is defined as

$$
S_{\epsilon}(\omega, \kappa)=\int c_{\epsilon}(\tau, \mathbf{y}) \exp \left[i\left(\kappa^{\prime} \mathbf{y}-\omega \tau\right)\right] d \mathbf{y},
$$

assuming that $c_{\epsilon}$ is absolutely integrable. The power spectrum of the noise is $S_{\epsilon}(\omega, 0)$.

Assuming that the channels are sampled at times $t_{j}=j \Delta$ for $j=0, \ldots, N-1$, define the discrete Fourier transform

$$
\left(\epsilon\left(\mathbf{x}_{k}\right)=\sum_{j=0}^{N-1} \epsilon(j \Delta) \exp (-i \omega j \Delta): k=1, \ldots, M\right) .
$$

If $S_{\epsilon}(\omega, 0)$ is bandlimited at $\pi / \Delta$, then $N^{-1} E\left|\epsilon\left(\mathbf{x}_{k}\right)\right|^{2}$ $\simeq \Delta^{-1} S_{\epsilon}(\omega, 0)$ for large $N .^{6}$ Let us work with the $\epsilon\left(\boldsymbol{x}_{\boldsymbol{k}}\right)$ to obtain an estimator of $S_{\epsilon}(\kappa, \omega)$ for a given $\omega$, which will be denoted $S_{\epsilon}(\kappa)$ to simplify notation. The properties of the estimator depend on the following theorem.

Theorem: Define the $n$-dimensional Fourier transform, ${ }^{7}$ 


$$
U(\kappa)=\sum_{k=1}^{k} \epsilon\left(\mathbf{x}_{k}\right) \exp \left(i \kappa^{\prime} \mathbf{x}_{k}\right)
$$

Assume that $D(\sigma)=\int f^{2}(\mathbf{x}) d \mathbf{x}=O\left(\sigma^{-n}\right)$ and when $\kappa \neq 0$, $|\phi(\kappa)|<c \sigma^{-n}$ for some constant $c$, where $\phi(\kappa)=E \exp \left(i \kappa^{\prime} \mathbf{X}_{k}\right)$ is the characteristic function of $\mathbf{X}_{k}$. These assumptions hold for the multivariate normal and uniform densities. Then

$$
\lim _{M, O \rightarrow \infty}\left(D M^{2}\right)^{-1} E|U(\kappa)|^{2}=S_{\epsilon}(\kappa),
$$

and $U\left(\kappa_{1}\right)$ and $U\left(\kappa_{2}\right)$ are asymptotically uncorrelated for $\kappa_{1} \neq \kappa_{2}$.

Proof: The array transfer function is

$$
R(\kappa)=\sum_{k=1}^{M} \exp \left(i \kappa^{\prime} \mathbf{x}_{k}\right) \text {. }
$$

For large $M, M^{-1} R(\kappa)=\phi(\kappa)+O_{p}\left(M^{-1 / 2}\right)$ by the central limit theorem. Thus

$$
\left(D M^{2}\right)^{-1} R\left(\kappa_{1}\right) R *\left(\kappa_{2}\right)=D^{-1} \phi\left(\kappa_{1}\right) \phi^{*}\left(\kappa_{2}\right)+O_{p}\left(M^{-1 / 2}\right)
$$

(star denotes complex conjugate) since $D^{-1}|\phi(\kappa)|=O(1)$ in the cross product by the above assumptions. Thus

$$
\begin{aligned}
\lim _{\mu \rightarrow \infty}\left(D M^{2}\right)^{-1}(2 \pi)^{-n} \int|R(\kappa)|^{2} d \kappa & =D^{-1}(2 \pi)^{-n} \int|\phi(\kappa)|^{2} d \kappa \\
& =D^{-1} \int f^{2}(\mathbf{x}) d \mathbf{x}=1 .
\end{aligned}
$$

From (10),

$$
\lim _{M \rightarrow \infty}\left(D M^{2}\right)^{-1}|R(0)|^{2}=D^{-1}|\phi(0)|^{2}=D^{-1}=O\left(\sigma^{n}\right) .
$$

Thus (11) implies that as $M$ and $\sigma \rightarrow \infty,\left(D M^{2}\right)^{-1}|R(\kappa)|^{2}$

$-\delta(\kappa)$, a Dirac delta function. If $\kappa_{1} \neq \kappa_{2}$,

$$
\left(D M^{2}\right)^{-1} R\left(\kappa_{1}\right) R *\left(\kappa_{2}\right)=O\left(\sigma^{-n}\right)+O_{p}\left(M^{-1 / 2}\right) \text {. }
$$

These limit results are used as follows:

$$
\begin{aligned}
E\left[U\left(\kappa_{1}\right) U^{*}\left(\kappa_{2}\right)\right]= & \sum_{=1}^{k} \sum_{k=1}^{N} c_{\epsilon}\left(\mathbf{x}_{j}-\mathbf{x}_{k}\right) \exp \left[i\left(\kappa_{1}^{\prime} \mathbf{x}_{j}-\kappa_{2}^{\prime} \mathbf{x}_{k}\right)\right] \\
= & (2 \pi)^{-n} \sum_{j=1}^{\mu} \sum_{k=1}^{n} \int S_{\epsilon}(\mathbf{v}) \exp \left[-i v\left(\mathbf{x}_{j}-\mathbf{x}_{k}\right)\right] \\
& \times \exp \left[i\left(\kappa_{1}^{\prime} \mathbf{x}_{j}-\kappa_{2}^{\prime} \mathbf{x}_{k}\right)\right] d v
\end{aligned}
$$

from the inverse of (9). Gathering terms,

$$
E\left[U\left(\kappa_{1}\right) U^{*}\left(\kappa_{2}\right)\right]=(2 \pi)^{-n} \int R\left(\kappa_{1}-\nabla\right) R *\left(\kappa_{2}-\nabla\right) S_{\epsilon}(\nabla) d v .
$$

Thus from the above limits and (14),

$$
\begin{aligned}
& \lim _{\boldsymbol{\nu}, a \rightarrow \infty}\left(D M^{2}\right)^{-1} E|U(\kappa)|^{2}=(2 \pi)^{-n} \int \delta(\kappa-\nabla) S_{\epsilon}(\nabla) d \nabla=S_{\epsilon}(\kappa) . \\
& \text { If } \kappa_{1} \neq \kappa_{2}, \text { then } \\
& \lim _{\mu, 0 \rightarrow \infty}\left(D M^{2}\right)^{-1} E\left[U\left(\kappa_{1}\right) U^{*}\left(\kappa_{2}\right)\right]=0
\end{aligned}
$$

from (12). Thus $U\left(\kappa_{1}\right)$ and $U\left(\kappa_{2}\right)$ are asymptotically uncorrelated. For finite $M \ll \sigma^{2 n}$, the correlation is $O\left(M^{-1 / 2}\right)$.

This theorem provides a basis for estimating $S_{\epsilon}(\kappa)$. One method is to divide the (time) sample into $J$ segments of successive observations, $N_{J}=N / J$, and compute $U(\kappa)$ for each segment. These $U_{f}(\kappa)$ 's will be approximately uncorrelated if $N_{j}$ is large. Thus from the theorem,

$$
\hat{S}_{\epsilon}(\kappa)=J^{-1} \sum_{j=1}^{J}\left(D M^{2}\right)^{-1}\left|U_{j}(\kappa)\right|^{2} \simeq S_{\epsilon}(\kappa),
$$

for large $J, M$, and $\sigma$. Since $U_{j}(\kappa)$ have a complex Gaussian distribution for each $j$ (the noise is Gaussian), $2\left(D M^{2}\right)^{-1}\left|U_{j}(\kappa)\right|^{2} / S_{\epsilon}(\kappa)$ is approximately chi-squared with two degrees of freedom and thus the variance of $\hat{S}_{\epsilon}(\kappa)$ is approximately $J^{-1} S_{\epsilon}^{2}(\kappa)$.

\section{A PLANAR ARRAY EXAMPLE}

Continuing with the vector notation, suppose that the sensors are uniformly distributed on the square $\left\{-L / 2 \leqslant x_{1} \leqslant L / 2,-L / 2 \leqslant x_{2} \leqslant L / 2\right\}$. Thus $f(x)=1 / L^{2}$ for $\mathrm{x}$ in the square, $\sigma_{1}^{2}=\sigma_{2}^{2}=\sigma^{2}=L^{2} / 12$, and $D=\int f^{2}(\mathbf{x}) d \mathbf{x}$ $=L^{-2}$. The assumptions for the theorem hold since $D=O\left(\sigma^{-2}\right)$ and $\phi(\kappa)=4\left(\kappa_{1} \kappa_{2} L^{2}\right)^{-1} \sin \left(\kappa_{1} L / 2\right) \sin \left(\kappa_{2} L / 2\right)$ $=O\left(\sigma^{-2}\right)$. Thus $(L / M)^{2} E|U(\kappa)|^{2} \simeq S_{\epsilon}(\kappa)$ for large $M$ and $L$ in this example. The estimator of $S_{\epsilon}(\kappa)$ is then

$$
(L / M)^{2} J^{-1} \sum_{j=1}^{J}\left|U_{j}(\kappa)\right|^{2}
$$

using the time segmentation method.

${ }^{1} \mathrm{~J}$. V. Thorn, N. Booth, and J. C. Lockwood, "Random and Partially Random Acoustic Arrays," J. Acoust. Soc. Am. 67, 1277-1285 (1980).

${ }^{2}$ B. D. Steinberg, Principles of Aperture and Array System Design (Wiley, New York, 1976), Chap. 8.

${ }^{3}$ M. J. Hinich, "Frequency-Wavenumber Array Processing," J. Acoust. Soc. Am. 69, 732-737 (1980).

${ }^{4}$ M. J. Levin, "Least-Squares Array Processing for Signals of Unknown Form," Radio Electron. Eng. 29, 213-222 (1965).

${ }^{5}$ Maximizing (8) to obtain the ML estimator of $\kappa$ follows from expressions (2.6) and (2.10) in M. J. Hinich and P. Shaman, "Parameter Estimation for an $r$-Dimensional Plane Wave Observed with Additive Independent Gaussian Errors," Ann. Math. Statist. 43, 153-169 (1972).

${ }^{6} \mathrm{D}$. Brillinger, Time Series, Data Analysis and Theory (Holt, Rinehart, and Winston, New York, 1975), Sec. 4.4.

${ }^{7}$ In practice the $x_{k}$ coordinates would be rounded to the nearest point on the $n$-dimensional grid $\left\{l_{1} d, \cdots l_{n} d\right\}$ where $d$ is a space unit and $l_{j}$ are integers. If we set $\epsilon\left(x_{k}\right)=0$ if there is no sensor at $x_{k}$ on the grid, then the FFT algorithm can be used to compute $U(x)$. 